\title{
PREVALÊNCIA DE SOBREPESO E OBESIDADE INFANTIL EM INSTITUIÇÕES DE ENSINO: PÚBLICAS VS. PRIVADAS
}

\author{
PREVALENCE OF OVERWEIGHT AND OBESITY IN CHILDHOOD IN EDUCATIONAL \\ INSTITUTIONS: PUBLIC VS PRIVATE
}

Artigo Original ORIGINAL ARTICLE Artículo Original

\author{
PREVALENCIA DE SOBREPESO Y OBESIDAD EN LA INFANCIA EN LAS INSTITUCIONES \\ EDUCATIVAS: PÚBLICAS VS. PRIVADAS
}

João Marcelo de Queiroz Miranda (Educador Físico)

Marcus Vinícius Palmeira ${ }^{3}$

(Educador Físico)

Luis Felipe Tubagi Polito'

(Educador Físico)

Maria Regina Ferreira Brandão (Psicóloga)

Danilo Sales Bocalini ${ }^{1}$

(Educador Físico)

Aylton José Figueira Junior ${ }^{1}$

(Educador Físico)

Kátia Ponciano ${ }^{1}$

(Educadora Física)

Rogério Brandão Wichi²

(Educador Físico)

1. Programa de pós graduação stricto sensu em Educação Física, Universidade São Judas Tadeu, São Paulo, SP, Brasil.

2. Universidade Federal do Sergipe, Aracajú, Brasil.

3. Universidade Federal de São

Paulo, São Paulo, Brasil.

\section{Correspondência:}

Kátia Ponciano. Programa de Pós-graduação Stricto Sensu em Educação Física, Universidade São Judas Tadeu. Av. Oratório 2635, apto 101, Torre 1 , Parque São Lucas. 03221-100, São Paulo, SP, Brasil. kcapristi@gmail.com

\section{RESUMO}

Introdução: dados epidemiológicos indicam que a obesidade infantil está aumentando na população brasileira e é apontada como um fator de risco para o desenvolvimento precoce de doenças crônicas. A obesidade tem sido caracterizada pelo aumento do tecido adiposo e do peso corporal. Objetivo: diagnosticar e comparar a prevalência de sobrepeso e obesidade em escolares da rede pública (EPU) e privada (EPR). Além disso, foi feita uma correlação entre o IMC e o percentual de gordura corporal em crianças de ambos os sexos. Métodos: foram avaliadas variáveis antropométricas (IMC e DOC) de meninos $(n=91)$ e meninas $(n=109)$ entre 8 e 10 anos de idade. Foi observada maior prevalência de sobrepeso em meninos (19,6\%) e meninas (25,5\%) da EPR em comparação com meninos $(6,7 \%)$ e meninas $(8,1 \%)$ da EPU. O nível de obesidade também foi maior em meninos $(34,8 \%)$ e meninas $(31,9 \%)$ da EPR quando comparado com meninos $(6,7 \%)$ e meninas $(6,5 \%)$ da EPU. Obteve-se correlação positiva entre as variáveis de IMC e percentual de gordura corporal de meninos ( $r=0,82)$ e meninas $(r=0,85)$. Resultados: os resultados sugerem que, apesar do aumento em todas as classes sociais, o sobrepeso e a obesidade mostram-se mais presentes naqueles com poder econômico maior, verificando-se, portanto, uma relação socioeconômica. Essa constatação pode levar a um estado de saúde mais próximo do polo negativo. Conclusão: a análise do IMC demonstrou ser um índice para determinação eficiente de sobrepeso e obesidade em crianças.

Palavras-chaves: obesidade, indicadores econômicos; índice de massa corporal.

\begin{abstract}
Introduction: epidemiological data indicate that childhood obesity is increasing in Brazil and is identified as a risk factor for early development of chronic diseases. Obesity has been characterized by increased adipose tissue and body weight. Objective: to diagnose and compare the prevalence of overweight and obesity in schoolchildren from public (EPU) and private (EPR) schools. Methods: anthropometrics variables (BMI and DOC [skin folds]) were evaluated in boys $(n=91)$ and girls $(n=109)$ between 8 and 10 years old. There was a higher prevalence of overweight in boys (19.6\%) and girls (25.5\%) of the EPR compared to boys (6.7\%) and girls (8.1\%) of the EPU. The obesity level was higher in boys (34.8\%) and girls (31.9\%) of the EPR compared to boys (6.7\%) and girls (6.5\%) of the EPU. Positive correlation was obtained between the variables BMI and \% body fat in boys $(r=0.82)$ and girls $(r=0.85)$. Results: the results suggest that despite the increase in all social classes, overweight and obesity prevalence were more present in those with higher economic status, confirming therefore a socioeconomic relationship. This finding may lead to a health condition closest to the negative pole. Conclusion: the BMl analysis proved to be an index for efficient determination of overweight and obesity in children.
\end{abstract}

Keywords: obesity, economic indicators; body mass index.

\section{RESUMEN}

Introducción: los datos epidemiológicos indican que la obesidad infantil se ha incrementado en Brasil y que se identifica como un factor de riesgo para el desarrollo temprano de las enfermedades crónicas. La obesidad se ha caracterizado por un aumento de tejido adiposo y del peso corporal. Objetivo: diagnosticar y comparar la prevalencia de sobrepeso y obesidad en escolares de instituciones educativas públicas (EPU) y privadas (EPR). Además, se encontró una correlación entre el IMC y el porcentaje de grasa corporal en niños de ambos sexos. Métodos: se evaluaron las variables antropométricas (IMC y DOC [pliegues cutáneos]) de niños ( $n=91)$ y niñas $(n=109)$ de entre 8 y 10 años de edad. Hubo una mayor prevalencia de sobrepeso en los niños (19,6\%) y niñas (25,5\%) de EPR en comparación con los niños (6,7\%) y niñas $(8,1 \%)$ de EPU. El nivel de obesidad fue mayor en los niños $(34,8 \%)$ y niñas $(31,9 \%)$ de EPR en comparación con los niños $(6,7 \%)$ y niñas $(6,5 \%)$ de EPU. Una correlación positiva se obtuvo entre las variables IMC y el porcentaje de grasa corporal de los niños $(r=0,82)$ y niñas $(r=0,85)$. Resultados: los resultados sugieren que, a pesar del aumento en todas las clases sociales, el sobrepeso y la obesidad eran más presentes en aquellos con mayor poder económico, por lo tanto existe una relación socioeconómica. Este hecho puede dar lugar a un estado de salud más cerca de un polo negativo. Conclusión: el análisis de IMC resultó ser un índice para la determinación eficiente de sobrepeso y obesidad en los niños.

Palabras clave: obesidad, indicadores económicos, indice de masa corporal. 


\section{INTRODUÇÃO}

O aumento da prevalência de obesidade infantil tem sido observado na população brasileira, e apontado como um fator de risco para o desenvolvimento precoce de doenças crônicas. A obesidade tem sido caracterizada pelo aumento do tecido adiposo e do peso corporal ${ }^{1-2}$. Esse distúrbio pode ter causa multifatorial, fazendo com que o tratamento da obesidade seja altamente complexo. Para alguns autores, o aumento do peso corporal pode ser decorrente de fatores hereditários e de fatores comportamentais ${ }^{3-5}$.

A obesidade pode iniciar em qualquer idade, desencadeada por fatores como o desmame precoce, má distribuição de alimentos durante a infância, substituição do aleitamento materno pelo consumo excessivo de carboidratos, bem como o sedentarismo ${ }^{2}$. Nesse sentido, estudos realizados nas cidades brasileiras têm demonstrado aumento da prevalência de sobrepeso e obesidade em crianças ${ }^{6,7}$ e demonstrado à associação com alguns fatores de risco como hipertensão arterial, hipercolesterolemia e resistência à insulina $a^{6-9}$. Entretanto, são poucos os estudos que levam em consideração a condição sócioeconômica como fator que pode contribuir no desenvolvimento do sobrepeso ou da obesidade.

O índice de massa corporal (IMC) tem sido utilizado por uma grande parte dos estudos populacionais para avaliação e classificação do sobrepeso e obesidade em crianças por ser uma técnica antropométrica de fácil interpretação e baixo custo. Já a análise do percentual de gordura, através da técnica de dobras cutâneas mostra-se eficiente por ser mais barata e prática quanto sua aplicabilidade. Porém, a sua utilização em obesos ainda é questionada, devido aos diferentes protocolos e a espessura das dobras. Dessa forma, é importante analisar se há alguma correlação entre tais técnicas, tendo em vista que poucos estudos fazem associação do IMC com o percentual de gordura corporal em crianças.

Portanto, o objetivo do presente estudo foi diagnosticar e comparar a prevalência de sobrepeso e obesidade em crianças de duas escolas da cidade de São Paulo, com características socioeconômicas diferentes.

\section{MATERIAIS E MÉTODOS}

Este estudo é do tipo transversal com escolha da amostra probabilística. Para o desenvolvimento dessa pesquisa, foram selecionadas 200 crianças com idade entre 8 a 10 anos, de ambos os sexos e residentes na cidade de São Paulo. As crianças matriculadas no ensino fundamental da escola pública (EPU) apresentavam menor condição sócioeconômica pela localização e comunidade de baixa renda. A escola privada (EPR) pela sua localização, maior renda foi caracterizada com nível socioeconômico mais alto. O Termo de Consentimento Livre e Esclarecido foi assinado pelos pais ou responsáveis das crianças envolvidas no estudo. O estudo foi aprovado pelo Comitê de Ética da Universidade São Judas Tadeu, SP, Brasil, pelo número de protocolo no 063/07.

Para determinação de sobrepeso, obesidade e composição corporal foram realizados os seguintes procedimentos: avaliação antropométrica e composição corporal; com o objetivo de calcular o IMC foram mensurados: a estatura $(\mathrm{m})$ e a massa corporal total $(\mathrm{kg})$ utilizando estadiômetro integrado a uma balança de marca Filizola ${ }^{\circledR}$ (BR). Este índice foi calculado através da divisão do peso pela estatura ao quadrado e foram adotados os critérios de classificação do National Center For Health Statistics Center For Disease Control and Prevention ${ }^{10}$ (NCHS. CDC, 2000) no qual, o sobrepeso foi definido a partir do IMC igual ou superior ao percentil 85 para sexo e idade e a obesidade com o IMC igual ou superior ao percentil 95 . O percentual de gordura corporal foi obtido utilizando a técnica duplamente indireta de dobras cutâneas (DOC). Foram medidas as pregas cutâneas da região triciptal, no ponto médio entre o acrômio e o olécrano, e subescapular, a dois centímetros abaixo do bordo inferior da escápula, com o uso de um adipômetro de marca Sanny ${ }^{\circledR}$ (BR) e pressão constante de $10 \mathrm{~g} / \mathrm{mm}^{2}$.
As medidas de espessura foram sempre realizadas no hemicorpo direito do avaliado, utilizando o dedo indicador e o polegar da mão esquerda para diferenciar o tecido adiposo subcutâneo do tecido muscular. Aproximadamente um centímetro abaixo do ponto de reparo, pinçado pelos dedos, foram introduzidas as pontas do compasso. As hastes do compasso foram colocadas perpendicularmente à superfície da pele no local da medida. Todas as medidas foram realizadas com o avaliado em posição ortostática e em repouso. Para o cálculo do percentual de gordura corporal foi utilizada a equação do protocolo específico para crianças e adolescentes propostos por Boileau et al. apud por Costa ${ }^{11}$. Para classificação do percentual de gordura foi utilizado Lohman apud por Pitanga ${ }^{12}$. Segundo este autor, até $6 \%, 7$ a 12\%, 13 a 18\%, 19 a 25\%, 26 a $32 \%$ e maior que $32 \%$ de gordura corporal correspondem respectivamente à classificação de muito baixo, baixo, normal, moderadamente alto, alto e muito alto para os meninos. Para as meninas, até $11 \%, 12$ a 15\%, 16 a $25 \%, 26$ a 30\%, 31 a 35\% e maior que $35 \%$ correspondem respectivamente a classificação de muito baixo, baixo, normal, moderadamente alto, alto e muito alto para os valores de gordura corporal.

A tendência de distribuição normal das variáveis foi testada pelo teste de Kolmogorov-Smirnov. Foi realizado o teste t de Student para estabelecer diferenças para o IMC e percentual de gordura corporal entre os sujeitos de escola pública e privada. Seus valores foram apresentados em média e desvio padrão. As variáveis antropométricas foram correlacionadas através do teste de Pearson, adotando nível de significância em $5 \%$. O programa Instat (versão 3.0) foi utilizado para a análise dos dados.

\section{RESULTADOS}

Participaram do estudo 107 (53,5\%) crianças da escola pública (EPU) sendo 45 (22,5\%) do sexo masculino e 62 (31\%) do sexo feminino. Na escola privada (EPR) 93 (46,5\%) crianças participaram da pesquisa sendo 46 (23\%) do sexo masculino e 47 (23,5\%) do sexo feminino, totalizando uma amostra total de 200 crianças avaliadas. De acordo com o IMC na EPU as crianças do sexo masculino foram classificadas como eutróficas (tabela 1). De maneira inversa, os resultados demonstraram maior índice de IMC na EPR. O grupo de meninos com idade de 8 anos foi classificado como obeso. Nas faixas etárias de 9 e 10 anos, os grupos foram caracterizados com sobrepeso.

A análise da composição corporal demonstrou que meninos da EPU apresentaram valores normais para o percentual de gordura corporal (tabela 2). Na escola privada, o percentual de gordura dos meninos foi maior quando comparado aos valores obtidos na escola pública para todas as idades. $\mathrm{Na}$ EPR, o percentual de gordura das crianças de 8, 9 e 10 anos apresentaram valores considerados altos e moderadamente altos, respectivamente.

Tabela 1. Características antropométricas em crianças do sexo masculino de escola pública (EPU) e privada (EPR).

\begin{tabular}{c|c|c|c|c|c}
\hline & \multicolumn{2}{|c|}{ EPU } & \multicolumn{2}{c|}{ EPR } & \\
\hline Idade & $\mathbf{n}$ & IMC & $\mathbf{n}$ & IMC & $\mathbf{p}$ \\
\hline 8 & 4 & $15,1 \pm 1,6$ & 6 & $21,8 \pm 4,8^{*}$ & 0.01 \\
\hline 9 & 24 & $16,1 \pm 2,8$ & 26 & $19,7 \pm 4,6^{*}$ & 0.001 \\
\hline 10 & 17 & $16,1 \pm 1,9$ & 14 & $19,9 \pm 3,2^{*}$ & 0.002 \\
\hline
\end{tabular}

Valores em média \pm desvio padrăo, após aplicação do test $t$ de studant, ${ }^{*} p<0,05$ vs EPU.

Tabela 2. Características da composição corporal em crianças do sexo masculino de escola pública (EPU) e privada (EPR).

\begin{tabular}{|c|c|c|c|c|c|}
\hline \multirow[b]{2}{*}{ Idade } & \multicolumn{2}{|r|}{ EPU } & \multicolumn{2}{|r|}{ EPR } & \multirow[b]{2}{*}{$p$} \\
\hline & $\mathrm{N}$ & $\% G$ & $\mathrm{n}$ & $\% G$ & \\
\hline 8 & 4 & $15,1 \pm 2,8$ & 6 & $28 \pm 7,5^{*}$ & 0.001 \\
\hline 9 & 24 & $15,2 \pm 7,0$ & 26 & $19,6 \pm 8,2^{*}$ & 0.02 \\
\hline 10 & 17 & $14,9 \pm 6,4$ & 14 & $22,2 \pm 8,2^{*}$ & 0.001 \\
\hline
\end{tabular}

Valores em média \pm desvio padrão, após aplicação do test t de studant, ${ }^{*} p<0,05$ vs EPU. 
Da mesma forma que encontramos nas crianças do sexo masculino, o grupo das meninas da EPU também foram classificadas de acordo com o IMC e percentual de gordura corporal como eutróficas (tabela 3) e normais (tabela 4), respectivamente. Na EPR, o valor do IMC foi maior do que o observado na EPU em todas as faixas etárias. As meninas de 8, 9 e 10 anos foram classificadas com sobrepeso. Porém, os valores de percentual de gordura corporal apresentaram-se dentro da normalidade no grupo com 8 anos de idade. Nos grupos de meninas de 9 e 10 anos da mesma escola, foram observados percentuais de gordura corporal moderadamente alto.

A análise percentual da classificação do IMC dos escolares de ambas as escolas demonstrou os seguintes resultados: Na EPU, 26,7\% dos meninos e $14,5 \%$ das meninas foram diagnosticados como subnutridos. $O$ índice de subnutrição foi menor na EPR, sendo 6,5\% nos meninos e $0 \%$ nas meninas. Na EPU, a maior parte da amostra foi classificada como eutrófica, sendo $60 \%$ dos meninos e $71 \%$ das meninas. Na EPR os meninos (39,1\%) e meninas $(42,6 \%)$ apresentaram valores menores de eutrofismo quando comparados com a EPU. No entanto, a prevalência de sobrepeso na EPR foi três vezes maior em relação à EPU. Na EPR $19,6 \%$ dos meninos e 25,5\% das meninas apresentaram sobrepeso. Na EPU, 7\% dos meninos e de 8,1\% das meninas apresentaram sobrepeso. Além disso, a quantidade de escolares obesos na EPR também foi superior quando comparada à EPU (34,8\% e 31,9\% vs. 6.7\% e 6,5\% para meninos e meninas, respectivamente).

Para ambos os sexos, independente da escola, foram verificados correlação positiva entre as variáveis IMC e massa gorda para os meninos $(r=0,82)$ e meninas ( $r=0,85)$ estudadas (figura 1 ).

Tabela 3. Características antropométricas em estudantes do sexo feminino de escola pública (EPU) e privada (EPR).

\begin{tabular}{c|c|c|c|c|c}
\hline & \multicolumn{2}{|c|}{ EPU } & \multicolumn{2}{c|}{ EPR } & \\
\hline Idade & $\mathbf{n}$ & IMC & $\mathbf{n}$ & IMC & $\mathbf{p}$ \\
\hline 8 & 11 & $15,7 \pm 2,8$ & 9 & $18,0 \pm 1,5^{*}$ & 0.02 \\
\hline 9 & 38 & $16,1 \pm 3,1$ & 20 & $20,4 \pm 3,8^{*}$ & 0.0001 \\
\hline 10 & 13 & $17,6 \pm 3,2$ & 18 & $19,7 \pm 3,1^{*}$ & 0.04 \\
\hline
\end{tabular}

Valores em média \pm desvio padrão, após aplicação do test $t$ de studant, $p<0,05$ vs EPU.

Tabela 4. Características da composição corporal em estudantes do sexo feminino de escola pública (EPU) e privada (EPR).

\begin{tabular}{|c|c|c|c|c|c|}
\hline \multirow[b]{2}{*}{ Idade } & \multicolumn{2}{|r|}{ EPU } & \multicolumn{2}{|r|}{ EPR } & \multirow[b]{2}{*}{$p$} \\
\hline & $\mathrm{n}$ & $\% G$ & $n$ & $\% G$ & \\
\hline 8 & 11 & $19 \pm 7,1$ & 9 & $23,4 \pm 2,5^{*}$ & 0.04 \\
\hline 9 & 38 & $20,2 \pm 5,3$ & 20 & $28,2 \pm 7,7^{*}$ & 0.0001 \\
\hline 10 & 13 & $21,6 \pm 4,5$ & 18 & $26,1 \pm 6,4^{*}$ & 0.01 \\
\hline
\end{tabular}

Valores em média \pm desvio padrão, após aplicação do test $t$ de studant, ${ }^{*} p<0,05$ vs EPU.

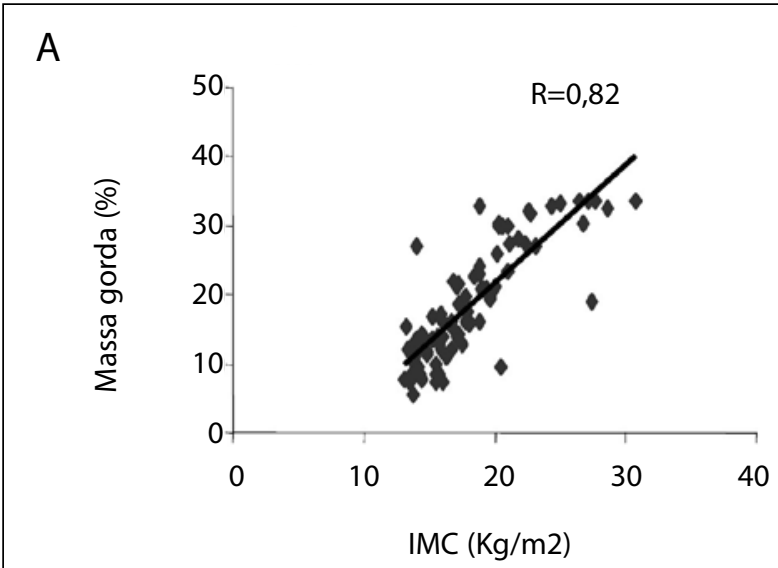

\section{DISCUSSÃO}

Os resultados encontrados demonstram que a prevalência de sobrepeso e obesidade infantil na escola privada é maior quando comparado aos da escola pública. Percentual mais alto de gordura corporal também foi observado nos estudantes da instituição privada. Foi evidenciado também que o aumento do peso e da gordura corporal ocorre já precocemente, principalmente nos meninos da escola privada. Além disso, o presente estudo evidenciou correlação positiva entre o IMC e o percentual de gordura corporal em crianças de ambos os sexos.

A utilização do IMC para diagnosticar sobrepeso, obesidade e também desnutrição, tem sido utilizado por diferentes autores ${ }^{12}$. Somado a sua simplicidade, este é um dos fatores que levam grande parte dos pesquisadores a utilizá-lo em seus estudos. Mas, segundo Monteiro e Fernandes Filho ${ }^{13}$ este índice não leva em consideração a composição proporcional do organismo o que se torna seu principal fator limitante se o objetivo for analisar a composição corporal do indivíduo. Em contrapartida a técnica de dobras cutâneas nos permite fracionar o peso corporal em dois componentes, a massa livre de gordura (MLG) e a massa de gordura (MG). No presente estudo verificou-se uma correlação positiva entre essas duas técnicas antropométricas demonstrando que, tanto para meninos $(r=0,82)$ quanto para meninas $(r=0,85)$, quanto maior for o IMC, maior também será o percentual de gordura corporal.

Oliveira e Veiga ${ }^{14}$ realizaram um estudo na cidade do Rio de Janeiro, com 303 adolescentes de escola pública e privada. Este estudo também verificou maior prevalência de sobrepeso e obesidade na escola privada, mas, os valores foram inferiores aos encontrados em nosso estudo. Apesar do estudo citado também ter utilizado as mesmas dobras cutâneas para estimar o percentual de gordura, eles adotaram outro critério de classificação para o IMC. Houve também uma amostra composta por adolescentes em um nível maturacional diferente, isto pode justificar os valores inferiores encontrados no nosso estudo. Outro estudo realizado por Leão et al. ${ }^{15}$ na cidade de Salvador/ Bahia foi observado maior prevalência de sobrepeso e obesidade nas escolas da rede privada, corroborando com o presente estudo. Nesse estudo, 387 crianças de 5 a 10 anos de idade foram avaliadas apenas pelo IMC, sem mensuração da composição corporal. Diniz et al. ${ }^{16}$ avaliou 694 escolares com idades de 8 a 11 anos de um município do estado do Rio Grande do Sul e verificou que 29,1\% de meninos e $32,6 \%$ das meninas apresentaram valores excessivos de gordura corporal. Esses valores se aproximam apenas dos valores encontrados nas meninas da escola privada do nosso estudo. Ricardo et al..$^{17}$ utilizando os critérios do Obesity Task Force (2000) avaliaram 322 escolares entre 6 e 14 anos de idade de escola pública e privada

Figura 1. Correlação entre massa gorda e IMC de escolares de 8 a 10 anos de idade: meninos (A) e meninas (B). 
e verificaram maiores índices de massa corporal na escola privada tanto para os meninos quanto para as meninas. 0 resultado deste estudo corrobora com os nossos achados.

Da mesma forma que Silva et al. ${ }^{7}$ não coletaram as variáveis necessárias para classificação mais precisa da condição sócioeconômica, atribuindo a isto uma limitação do presente estudo. Porém, corroboramos com o estudo de Silva de que as crianças de maior renda frequentam as escolas privadas e crianças que frequentam o serviço público educacional possuem menor renda. Dessa forma, os resultados obtidos no presente estudo sugerem que crianças de famílias com maior renda possuem mais acesso a alimentos de maior densidade energética, resultando num balanço energético positivo. Além disso, a possibilidade de maior acesso à tecnologia pelas crianças da escola privada pode gerar menor nível de atividade física nos momentos de lazer, quando comparados às crianças de menor renda. Um estudo realizado por Zago Júnior et $a l^{18}{ }^{18}$ em crianças com 6 a 11 anos de uma escola particular da cidade de Campinas, a prevalência de sobrepeso (10\%) e obesidade (17,5\%) foi relacionado com o tempo médio de 4,8 horas por dia em frente à televisão. Para Leão et al. ${ }^{15}$ o fácil acesso ao consumo de alimentos ricos em calorias vazias e diminuição da ativi- dade física são hábitos presentes principalmente em crianças de maior nível socioeconômico levando ao sedentarismo precoce.

\section{CONCLUSÕES}

Os resultados do presente estudo sugerem que o desenvolvimento de sobrepeso e obesidade infantil está relacionado ao fator socioeconômico, podendo por sua vez, levar à um estado mais próximo de um polo negativo de saúde podendo favorecer precocemente o desenvolvimento de doenças crônicas. Além disso, pode-se concluir que o IMC é um importante índice para diagnosticar o estado nutricional de crianças, correlacionando-se positivamente com o percentual de gordura. Dessa forma, o desenvolvimento de estratégias de intervenção pode ser útil para prevenção da obesidade infantil. Serão necessários outros estudos que investiguem o nível de atividade física e o padrão alimentar de escolares a fim de esclarecer a relação de causa/efeito do sobrepeso e obesidade em crianças.

Todos os autores declararam não haver qualquer potencial conflito de interesses referente a este artigo.

\section{REFERÊNCIAS}

1. Singulem DM, Taddei JAAC, Escrivão MAM, Devincenzi UM. Obesidade na infância e na adolescência. São Paulo: Planejamento Editorial: EPM - Editora de Projetos Médicos; 2000

2. Giugliano R, Carneiro EC. Factors associated with obesity in school children. J Pediatr. 2004;80(1):17-22

3. Carneiro JRI, Kushnir MC, Clemente ELS, Brandão MG, Gomes MB. Obesidade na adolescência: fator de risco para complicações clínico-metabólicas. Arq Bras Endocrinol Metab. 2000;44(5):390-6.

4. Ramos AM, Barros Filho AA. Prevalência da obesidade em adolescentes de bragança paulista e sua relação com a obesidade dos pais. Arq Bras Endocrinol Metab. 2003:47(6):663-8.

5. Luiz AMA, Gorayeb R, Liberatore Júnior RDR, Domingos NAM. Depressão, ansiedade e competência social em crianças obesas. Estud Psicol (Natal). 2005;10(1):35-9.

6. Soar C, Vasconcelos FAG, Assis MAA. Prevalência de sobrepeso e obesidade em escolares de uma escola pública de Florianópolis, Santa Catarina. Rev Bras Saude Mater Infant. 2004;4(4):391-7.

7. Silva GAP, Balaban G, Motta MEF. Prevalência de sobrepeso e obesidade em crianças e adolescentes de diferentes condições socioeconômicas. Rev Bras Saude Mater Infant. 2005;5(1):53-9.

8. Parente EB, Guazzelli I, Ribeiro MM, Silva AG, Halpern A, Vilaares SM. Perfil lipídico em crianças obesas: efeitos de dieta hipocalórica e atividade física aeróbica. Arq Bras Endocrinol Metab. 2006:50(3):499-504

9. Ribeiro RQC, Lotufo PA, Lamounier JA, Oliveira RG, Soares JF, Botter DA. Fatores adicionais de risco cardiovascular associados ao excesso de peso em crianças e adolescentes. 0 estudo do coração de Belo Horizonte. Arq Bras Cardiol. 2006;86(6):408-418.

10. Nacional Center Health Statistic. CDC About body mass index for childrem and teens. Disponível em: <http://www.cdc.gov. [Acesso em: 22/Agosto/2007].

11. Costa RF. Composição corporal: teoria e prática da avaliação. São Paulo: Manole; 2001

12. Pitanga FJ. Testes, medidas e avaliação em educação física e esportes. São Paulo: Phorte; 2005.

13. Monteiro AB, Fernandes Filho J. Análise da composição corporal: uma revisão de métodos. Rev Bras Cineantropom Desempenho Hum. 2002;4(1):80-92.

14. Oliveira CS, Veiga GV. Estado nutricional e maturação sexual de adolescentes de uma escola pública e de uma escola privada do município do Rio de Janeiro. Rev Nutr. 2005;18(2):183-91.

15. Leão LS, Araújo LM, Moraes LT, Assis AM. Prevalência de obesidade em escolares de Salvador, Bahia. Arq Bras Endocrinol Metab. 2003;7(2):151-157.

16. Diniz IMS, Lopez AS, Dummel CCB, Rieger T. Crescimento físico e adiposidade corporal de escolares. Rev Bras Cineantropom Desempenho Hum. 2006;8(2):32-38

17. Ricardo GD, Zolet KE, Monte FG, Quites MP, Carvalho T. Prevalências de sobrepeso e de obesidade em estudantes de escola pública e privada de florianópolis, SC. Rev Bras Ativ Fis Saúde. 2007;12(2):162.

18. Zago Júnior C, Rodrigues AA, Braga LES, Filocomo M, Zamai C. Perfil nutricional de crianças de 6 a 11 anos de uma escola. Rev Bras Ativ Fis Saúde. 2007;12(2):159. 